\title{
Modified Biperiodic Structures for Linear Particle Accelerators
}

\author{
Vladimir Kuzmich Shilov ${ }^{1}$, Aleksandr Nikolaevich Filatov ${ }^{2}$, Aleksandr Evgenevich Novozhilov ${ }^{3}$ \\ ${ }^{1}$ National Research Nuclear University MEPhI (Moscow Engineering Physics Institute), Moscow, Russian Federation \\ ${ }^{2}$ National Research Nuclear University MEPhI (Moscow Engineering Physics Institute), Moscow, Russian Federation \\ ${ }^{3}$ National Research Nuclear University MEPhI (Moscow Engineering Physics Institute), Moscow, Russian Federation
}

\begin{abstract}
This article discusses the properties of modified biperiodic structures with standing wave and improved focusing properties for effective electron acceleration in linear accelerators. Main attention is paid to possible application of the focusing properties of electromagnetic accelerating field for transmission of electron beam through aperture of biperiodic decelerating system, which would reduce significantly accelerator dimensions. Predictions of particle dynamics in standing wave accelerators based on biperiodic decelerating structures are presented, which confirms possibility of the beam transmission without application of external focusing elements. The discussed procedure can be applied for designing and development of standing wave linear particle accelerators.
\end{abstract}

Key words: linear particle accelerator, biperiodic decelerating system, standing wave, structure acceptance, beam emittance, drift bushing, steady radial motion range, acceleration range, area of drift channel, particle dynamics, aperture of drift channel, cell electromagnetic field.

\section{INTRODUCTION}

Application of linear particles accelerators (linac) in medicine and industry is attributed to their numerous advantages: simplicity of input and output of accelerated electrons, simplicity of adjustment of dose energy and rate, high dose rate of braking radiation even at comparatively moderate energies of accelerated particles.

Intensive development of accelerators for fundamental studies in the field of high energy physics and significant R\&D advantages in other fields provide possibility of practical use of accelerators in industry [1], medicine [2], and environmental protection [3].

In the energy range of $2-50 \mathrm{MeV}$, travelling wave accelerators are mostly used. However, when a compact accelerator is required, the standing wave accelerator is more preferred. Linac operation modes are compared in [4], reasonability of standing wave accelerators for low and medium energies is demonstrated.
Biperiodic decelerating structure (BDS) in standing wave linac can be considered as a chain of accelerating resonators located on one axis and interconnected by coupling cells [5]. Since the coupling cells are free from electromagnetic fields, then they can be located beyond the accelerating structure. Oscillations of electromagnetic fields in neighboring accelerating resonators differ in phase by $\pi$, hence, a particle should transit the distance between the centers of neighboring resonators in the time equaling to one half of period of electromagnetic oscillations. Only in this way the particle and accelerating field can be synchronized.

If the function of distribution of electric field intensity along the axis of standing wave linac is considered at certain time, then it can be seen that it is comprised of alternated pulses of different polarity with spatial period equaling to the generator wavelength [6]. The most promising in standing wave linac is focusing upon which beam transmission is based on focusing action of the electromagnetic field itself [7]. As mentioned above, this becomes possible due to occurrence of radial component of electric field in BDS cells [8]. Application of focusing properties of BDS of high frequency (HF) eigenfields reduces significantly transversal dimensions of accelerating section as well as total weight of accelerator. In addition, no huge power sources are required for external focusing devices adding difficulties in operation of accelerators, which is especially important in geology, medicine, and nondestructing testing.

\section{PROPOSED METHODOLOGY}

\subsection{General description}

Effective focusing in standing wave linac and accelerator efficiency are mainly determined by output beam quality and accelerated current.

The issues of focusing of accelerated electron beams by HF fields in standing wave linac with BDS based accelerating system are discussed in [9]. The advantages of this focusing method are obvious: refusal to use conventional focusing elements and their power sources leads to significant reduction of dimensions and weight of assembly, to improve mobility of radiating source. 


\subsection{Algorithm}

The focusing mechanism was initially analyzed by simplified model of BDS accelerating resonator. This model provided qualitatively correct presentation of occurring processes, confirmed by numerical predictions using software integrating equations of accelerated particles travelling in $\mathrm{HF}$ fields of cavity resonators. Using the analysis based on the proposed approach, the focusing properties of BDS with high acceleration rate (up to $58.3 \mathrm{MV} / \mathrm{m}$ for $10 \mathrm{~cm}$ range) were improved [10].

Let us analyze the action of HF focusing which is characterized by certain features in the considered case. In order to obtain visual physical pattern, let us apply the mathematical model of cell proposed in [9] with the only difference that its longitudinal size is two times lower. The computation result is that if accelerating field varies in time according to cosine law, then all regions of initial phases of electron entry, for which the conditions of acceleration and focusing by respective components of HF field are met, will be shifted by $\pi / 4$ in comparison with similar regions for conventional BDS.Therefore, in the case of accelerating cells with or without drift bushings in the form of regular cylindrical resonator, the phase regions of simultaneous acceleration and focusing will be as follows: for the cells of the first type:

$$
-\pi / 4 \pm 2 \pi n<\varphi_{0}<\pi / 4 \pm 2 \pi n
$$

where: $n=0,1,2, \ldots$, and $\varphi_{0}$ is the phase of HF field corresponding to particle entry into accelerating resonator; and for the cells of the second type:

$$
-3 \pi / 4 \pm 2 \pi n<\varphi_{0}<-\pi / 4 \pm 2 \pi n
$$

It could be mentioned that the length of phase regions, as before, is $\pi / 2$.

\subsection{Flow chart}

Designs and properties of modified accelerating structure of this type with improved focusing properties are considered and analyzed. These properties are achieved by manufacturing of the accelerating cells with one drift bushing (Fig. 1) with the conical drift orifice which modifies spatial configuration of $\mathrm{HF}$ fields in the system leading to variation of boundaries of respective phase regions of acceleration and focusing.
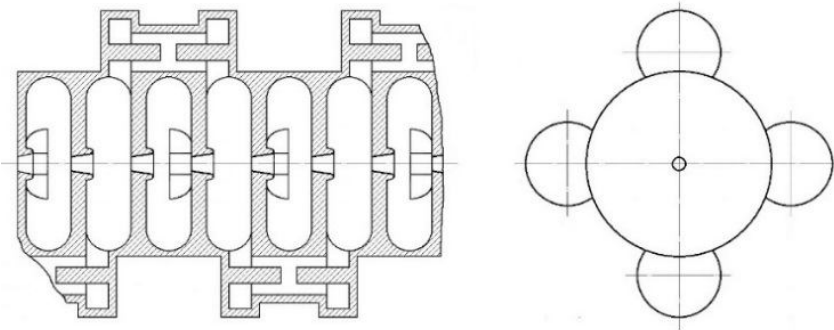

Figure 1: Modified BDS with high acceleration rate and significantly increased focusing action of HF eigenfields of accelerating cells.
In our case, without serious damage for final results, it is possible to neglect variation of distribution form of azimuthal magnetic and longitudinal electric fields, considering only for significant distribution of radial component of electric field. Then, for electron beam entering the accelerating cell from drift bushing, the region of focusing by radial electric filed will coincide with respective region of conventional BDS, and the region of simultaneous acceleration and focusing will be as follows $-\pi / 2 \pm 2 \pi n<\varphi_{0}<\pi / 4 \pm 2 \pi n$, moreover, the regions of focusing, due to radial component of electric fields and azimuthal component of magnetic field, are overlapped in the range of $-\pi / 2 \pm 2 \pi n<\varphi_{0}<-\pi / 4 \pm 2 \pi n$.

Therefore, the dimensions of region of initial electron entry, for which the conditions of simultaneous acceleration and focusing are met, increase by $50 \%$; moreover, the phase corresponding to maximum acceleration $(\pi / 4)$ is in the range of focusing region, enabling the regime providing both radial focusing of electron clusters and their optimum acceleration. Such favorable operation regime is unreachable in the case of BDS with regular accelerating cells.

More detailed information about focusing properties of both structures was obtained using software integrating equations of charged particles travelling in standing wave structures. HF fields interacting with accelerated electrons along overall accelerator length were preset every time in table form. Accelerating system was considered comprised of 16 identical accelerating cells with the longitudinal size corresponding to phase velocity equaling to $\beta$ (10 $\mathrm{cm}$ range), and the intensity of longitudinal electric field in the axis of accelerating cells was $180 \mathrm{kV} / \mathrm{cm}$. The phases were measured at the scale when 1 corresponded to $2 \pi$. The particles were in the structure with the initial radius $\rho=1 \mathrm{~mm}$, with the longitudinal initial relative velocity $\beta=0.97$ and relative radial velocity $\beta_{\rho}=0.01$.

\section{RESULTS AND DICSUSSION}

The predictions evidence that the focusing action on the accelerated electrons of modified HF structure is significantly higher. Correct comparison of focusing properties of regular BDS of the considered type and BDS with modified profile of accelerating cells was possible using such property as acceptance [11], which is the maximum possible emittance of matched beam still passing through the system. The higher is the acceptance, the higher is the capacity of HF structure. Figure 2 illustrates the acceptances for various initial phases of electron entry into the considered systems having the same apertures equaling to $10 \mathrm{~cm}$ 


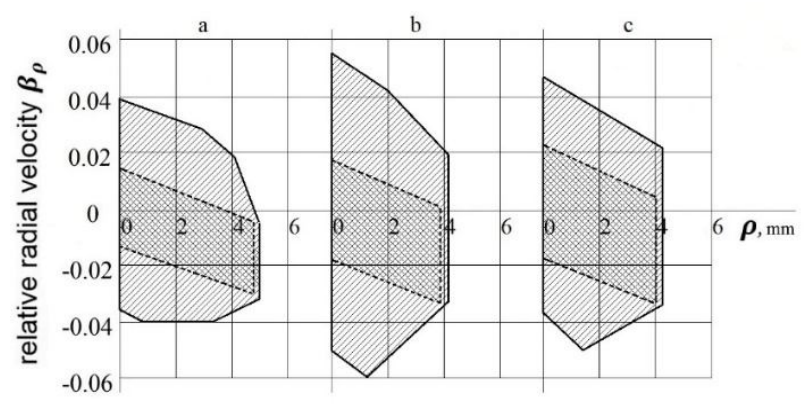

Figure 2: Acceptances for various initial stages of electron entry into accelerating systems: a) - phase $0.7, b$ ) - phase 0.8 ,

c) - phase 0.9; dashed line - acceptances of regular BDS, solid line - acceptances of modified BDS.

It can be seen in Fig. 2 that variation of form of accelerating cells by the mentioned method makes it possible to increase by two times and higher the phase surface area restricted by acceptances corresponding to maximum output energy gain by particles (phases $0.7-0.9$ ).

Therefore, the proposed modification of form of BDS accelerating cells is characterized by certain obvious advantages and allows to increase significantly the system efficiency in such promising regime as focusing of accelerated beam by HF eigenfields of accelerating structure [12].

For systems with coupling cells located on the axis of accelerating system some design approaches are available which improve focusing properties of such structures. Let us analyze separately the impacts of magnetic azimuthal $H_{\varphi}$ and electric radial $E_{\rho}$ components of electromagnetic HF field of resonator on accelerated electrons. The phase regions of simultaneous acceleration and focusing are either

$$
-\pi / 2 \pm 2 \pi n<\varphi_{0}<0 \pm 2 \pi n
$$

or

$$
-\pi \pm 2 \pi n<\varphi_{0}<-\pi / 2 \pm 2 \pi n
$$

where $n=0,1,2, \ldots$

Equation (3) corresponds to conventional $\Omega$ shaped BDS with optimized in terms of shunt resistance form of accelerating resonators. In this structure radial dynamics of electrons are more affected by electric radial component $E_{\rho}$. Equation (4) corresponds to BDS where accelerating resonators are regular unshaped cylindrical resonators, in this structure more significant influence on radial dynamics of electrons is exerted by magnetic azimuthal component $H_{\varphi}$.

Focusing action of standing wave BDS on electron beam can be more effective as a consequence of substitution of some shaped accelerating resonators with cylindrical unshaped resonators. The mechanism of HF focusing is also analyzed well by the proposed approach.

Figure 3 illustrates BDS with alternating cylindrical resonators of shaped and unshaped type. Herewith, for the phase region with simultaneous acceleration and focusing, one half of the resonators is described by Eq. (3), and the other half - by Eq. (4). Conic drift orifices in shaped accelerating resonators and cylindrical drift orifices of lower diameter in unshaped accelerating resonators make it possible to enhance focusing action on electrons in some resonators by radial component $E_{\rho}$, and in other resonators - by azimuthal component $H_{\varphi}$.
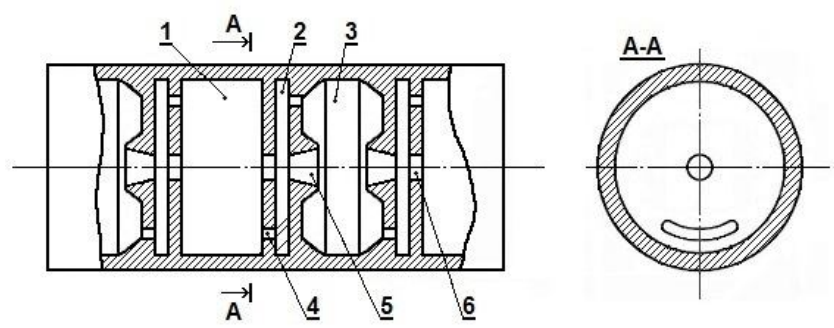

Figure 3: Standing wave linac with improved focusing properties: 1 - unshaped cylindrical accelerating resonator, 2 - cylindrical coupling resonator, 3 - shaped cylindrical accelerating resonator, 4 - azimuthal coupling slot, 5 - conic drift orifice, 6 - cylindrical drift orifice

Therefore, alternation of such accelerating resonators creates for the region of initial phases of electron entry (3) consecutively alternating focusing and defocusing segments, and for the phase region (4) it will be the sequence of defocusing and focusing segments. There exists somewhat similar alternative focusing in accelerators with the only difference that in this case focusing and defocusing are performed by alternatively varying azimuthal and electrical radial components of $\mathrm{BDS}$ of $\mathrm{HF}$ eigenfield.

The focusing period is twice as much as the period of electron acceleration and is comprised of two accelerating resonators of different profiles and two coupling resonators. As in the case of alternative focusing, cumulative focusing effect is achieved which is valued for overall phase interval of acceleration:

$$
-\pi \pm 2 \pi n<\varphi_{0}<0 \pm 2 \pi n .
$$

It should be mentioned that the phase $-\pi / 2$, corresponding to the regime of maximum energy gain by electrons in such structure, is in the center of HF focusing with the regime of optimum output energy gain by accelerated particles.

Possibilities of the proposed designing solutions improving focusing capabilities of BDS are exemplified by linac with replaceable bunchers. Each separate buncher contains two accelerating resonators, and their combination together with accelerating system itself (six accelerating resonator) provides accelerator operation both in the regime of 
maximum energy gain by electrons at output of accelerating system and in the regime with maximum coefficient of particle capture in acceleration at acceptable spectral properties of beam.

In rated regime at power source capacity of $1.4 M W(10 \mathrm{~cm}$ range) at accelerator output the pulse current of accelerated electrons is $200 \mathrm{~mA}$ at output energy of $3.2 \mathrm{MeV}$. The external diameter of linac HF structure is $90 \mathrm{~mm}$, the diameter of drift channel is $10 \mathrm{~mm}$, and the length of accelerating system is $45 \mathrm{~cm}$. The structure was considered where four, two, and one resonator of accelerating resonators, respectively, including accelerating resonator of buncher, were unshaped.

Comparison of acceptances of various structures demonstrated that application of even one-two unshaped resonators in combination with shaped resonators provided positive effect in terms of focusing: the surface area of transversal acceptances increased by 30-60\%, which corresponded to the phases where maximum electron acceleration took place. The results are illustrated in Fig. 4.

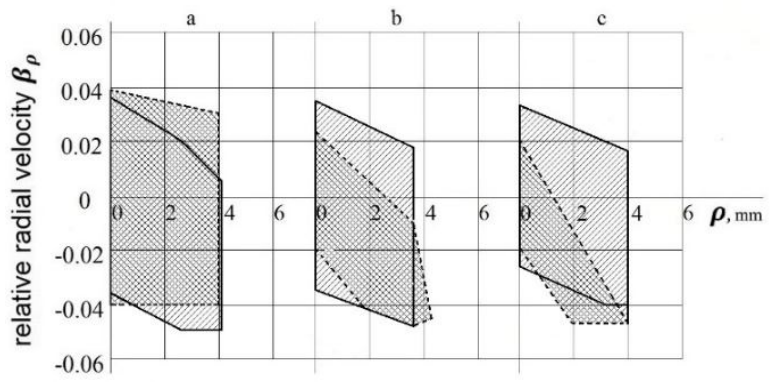

Figure 4: Acceptances for various initial phases of electron entry into accelerating systems: a) - phase $0.6, b$ ) - phase 0.7 ,

c) - phase 0.8; dashed line - acceptances of regular BDS, solid line - acceptances of modified BDS.

The experimental results completely confirmed the conclusion of simplified mathematical model of accelerating cell. The simplified model presents correct physical pattern of occurring processes, hence, it would be reasonable to apply this model for rapid determination of focusing properties of various HF standing wave structures.

An excellent style manual and source of information for science writers is [9].

\section{CONCLUSION}

Therefore, the obtained results completely confirm the conclusions of simplified mathematical model of accelerating cell and provide visual presentation of the occurring processes. As a consequence, we suppose to use it for rapid determination of focusing properties of HF standing wave structures.

The proposed method of transmission of electron beam in linear accelerators is based on the use of focusing properties of radial component of electric field. This component appears even at the lowest level of oscillations in regular cylindrical resonator in the range of drift tubes and, hence, near the drift channel axis. Such effect occurring due to curving of power lines of electromagnetic field can be promising for proton or ion linear accelerators $[13,14]$.

The studies based on mathematical simulation demonstrated possibilities of this procedures upon development and designing of linac without external focusing devices. This would allow to reduce significantly both dimension and weight of accelerator as well as to improve its operation efficiency in industrial fields and medicine.

It is planned to investigate into the influence of variations of BDS resonator shape on distribution of components of electric field in the vicinity of drift channel and conditions of effective acceleration and steady beam radial stability with accounting for spatial charge forces [15].

\section{ACKNOWLEDGEMENT}

This work was supported by MEPhI Academic Excellence Project (contract No. 02.a03.21.0005, 27.08.2013).

\section{REFERENCES}

1. B. Bogdanovich, V. Kaminskiy, A. Nesterovich, and V. Senyukov. Small-Sized Electron Linear RF Accelerator with Beam Auto Acceleration for Geology and Industry. Bulletin of American Physical Society, Vol. 3(42), pp. 1376, 1997.

2. L. Auditore, R.C. Barna, D. De Pasquale, U. Emanuele, A. Trifiro, and M. Trimarchi. A Compact $5 \mathrm{MeV}$, S-Band, Electron Linac Based X-Ray Tomography System. In 10th European Particle Accelerator Conference, Edinburgh, UK, 26-30 June 2006.

3. Y. Vakhrushin, G. Vyaz'mentzova, V. Kuznetzov, and R. Fidel'skaya. Dezinfektsiya stochnykh vod infektsionnykh bol'nits elektronnym puchkom, In Tezisy dokladov 8 Soveshchaniya po primeneniyu uskoriteley zaryazhennykh chastits v promyshlennosti i meditsine "Uskoriteli-95", Saint-Petersburg, 1995.

4. A. Zavadtsev. Biperiodic U-structures for Particle Accelerators. Fourth European Accelerator Conference, Vol. 3, pp. 2176-2178, 1994.

5. A.E. Novozhilov. Assessment of method errors in measurement of acceleration fields in accelerating sections of charged particle accelerators. Life Science Journal, Vol. 11(11s), pp. 506-510, 2014.

6. A.E. Novozhilov. Calculation of Resonant Frequencies and Electromagnetic Fields in Resonators of Linear Accelerators for Commercial Application, Medicine and Environmental Protection. Research Journal of Pharmaceutical, Biological and Chemical Sciences, Vol. 7(2), pp. 897-905, 2016.

7. A.E. Novozhilov. Distribution of Accelerating Voltage in Resonator of Linear Electron-Positron Collider. 
International Journal of Applied Engineering Research, Vol. 11(3), pp. 1596-1602, 2016.

8. A.N. Filatov, V.K. Shilov. Control of radio-frequency characteristics of linear electron accelerator. Instruments and Experimental Techniques New York, Vol. 28(6), pp. 1258-1261, November 1985.

9. A.E. Novozhilov. Problems of measurement of high-frequency fields in linear electron accelerators. Global Journal of Pure and Applied Mathematics, Vol. 12(1), pp. 643-655, 2016.

10. V.A. Vaguine, (1977). Standing Wave High Gradient Accelerator Structure. IEEE Transactions on Nuclear Science, Vol. 24(3), 1084-1086. https://doi.org/10.1109/TNS.1977.4328856

11. I.M. Kapchinsky. Theory of linear resonant accelerators. Moscow: Energoizdat, 1982.

12. A.E. Novozhilov, A.N.Filatov, V.K. Shilov. Calculation of bunchers in linear electron accelerators with standing wave. ARPN Journal of Engineering and Applied Sciences, Vol. 12(1), pp. 182-187. (2017)

13. S. Vdovin, P. Demchenko, E.Gusev, N. Shulika, and O. Shulika. Kombinirovannaya fokusirovka $\mathbf{v}$ lineynom uskoritele ionov [Combined Focusing in Linear Ion Accelerator]. Voprosy atomnoy nauki i tekhniki, Vol. 4(7), pp. 325-329, 2010.

14. V.E. Kalyuzhnyi. Relative Jitter of Accelerating Voltage Amplitude on Resonator's Cells of Linear Electron-positron collider TESLA-ILC. World Applied Sciences Journal, Vol. 27(12), pp. 1620-1624, 2013.

15. A.E. Novozhilov. Taking into Account Forces of Beam Spatial Charge upon Calculations of Particle Dynamics in Standing-Wave Accelerators. Research Journal of Pharmaceutical, Biological and Chemical Sciences, Vol. 7(4), pp.1552-1559, 2016. 